\title{
Occipital Nerve Stimulation in Chronic Migraine: The Relationship Between Perceived Sensory Quality, Perceived Sensory Location, and Clinical Efficacy-A Prospective, Observational, Non-Interventional Study
}

\author{
Carl H. Göbel (D) · Anna Göbel • Uwe Niederberger · Axel Heinze · Katja Heinze-Kuhn • \\ Christoph Meinecke · Hubertus M. Mehdorn · Dirk Rasche · Hartmut Göbel
}

Received: July 23, 2020 / Published online: September 10, 2020

(C) The Author(s) 2020

\begin{abstract}
Introduction: Occipital nerve stimulation (ONS) is used to treat therapy-resistant chronic migraine. Clinical use has resulted in a wide intraindividual and interindividual variation of clinical efficacy. The aim of this study was to analyze a potential relationship between sociodemographic variables, headache parameters, perceived sensory quality, perceived sensory location, as well as clinical efficacy.
\end{abstract}

Digital Features To view digital features for this article go to https://doi.org/10.6084/m9.figshare.12859901.

C. H. Göbel · A. Göbel · A. Heinze · K. Heinze-Kuhn . C. Meinecke · H. Göbel

Kiel Migraine and Headache Centre, Kiel, Germany

C. H. Göbel $(\varangle)$ · A. Göbel

Department of Neurology, University Hospital

Schleswig-Holstein, Campus Lübeck, Lübeck,

Germany

e-mail: carl.goebel@neuro.uni-luebeck.de

\section{U. Niederberger}

Institute of Medical Psychology and Medical

Sociology, University Hospital Schleswig-Holstein, Campus Kiel, Kiel, Germany

H. M. Mehdorn

Department of Neurosurgery, University Hospital Schleswig-Holstein, Campus Kiel, Kiel, Germany

\section{Rasche}

Department of Neurosurgery, University Hospital Schleswig-Holstein, Campus Lübeck, Lübeck, Germany
Methods: Thirty-two subjects $(21.9 \%$ male, mean age 45.77 years) suffering from chronic migraine refractory to other treatment and therefore treated with ONS were included in this study. We used a computer-based imaging method for mapping the ONS-induced perceived sensory location, the perceived spatial sensory field size, as well as the perceived sensory quality in a long-term course over 21 months in weekly time intervals. Additionally, the effect of ONS on the migraine headache was documented weekly by the participants using a verbal rating scale. Over the observation period, a total of 808 individual weekly data sets were recorded and a potential relationship between ONS-induced perceptions and headache parameters could be analyzed.

Results: We found that $48.9 \%$ of stimulation intervals were reported as effective by patients. Women displayed a significantly higher responder rate than men. The reported effectiveness did not differ depending on age, the average number of migraine days per month, the MIDAS score, or the duration of the migraine disorder prior to ONS treatment. Implantation with trial period led to significantly lower responder rates than without the trial period. The most frequently perceived sensory quality of "tingling" was found significantly more frequently in non-responders than in responders. Responders displayed significantly lower pleasantness scores for their reported perceptions than non-responders. 
Sensations that were spatially perceived above the line connecting the external acoustic meati with the external occipital protuberance (MOP line) led to patients reporting a positive clinical effect significantly more frequently than sensations spatially perceived below the MOP line. Spatially small fields of sensory perception were correlated with a higher responder rate than those covering broader areas.

Conclusions: The ONS-induced sensory location, the size of the spatial sensory field, as well as the sensory quality are significantly correlated with the reported clinical effectiveness. The results suggest that besides surgical technique, the individual and continuous programming of the stimulation parameters is clinically relevant in increasing the therapeutic effectiveness.

Keywords: Field of perception; Migraine; Neuromodulation; Occipital nerve stimulation; Programming; Supra-threshold sensory perception; Trial phase; Quality of perception

\section{Key Summary Points}

Why carry out this study?

Occipital nerve stimulation (ONS) is used to treat therapy-resistant chronic migraine.

Clinical use has resulted in a wide intraindividual and interindividual variation of clinical efficacy.

The aim of this study was to analyze a potential relationship between sociodemographic variables, headache parameters, perceived sensory quality, perceived sensory location, as well as clinical efficacy.

\section{What was learned from the study?}

The ONS-induced sensory location, the size of the spatial sensory field, as well as the sensory quality are significantly correlated with the reported clinical effectiveness.
The results suggest that, besides surgical technique, the individual and continuous programming of the stimulation parameters is clinically relevant in increasing the therapeutic effectiveness.

\section{DIGITAL FEATURES}

This article is published with digital features to facilitate understanding of the article. To view digital features for this article go to https://doi. org/10.6084/m9.figshare.12859901.

\section{INTRODUCTION}

Occipital nerve stimulation (ONS) is used to treat therapy-resistant chronic migraine, cluster headache, and other severe pain disorders [1-26]. Electrical impulses are generated by an implantable pulse generator (IPG), which is implanted usually in the lower back, abdomen, or chest. The electrical impulses are transmitted via subcutaneously implanted electrodes to the cutaneous innervation area of the occipital nerve. Occipital nerve stimulation induces sensory experiences (i.e., prickling, tingling, pinching) in the area, where the leads are surgically placed. It is assumed that ONS activates antinociceptive mechanisms that prevent the development of chronic pain [27-30]. However, no standardized surgery, lead localization, or programming has yet to be established. There is a wide intraindividual and interindividual variation of clinical efficacy. What this is caused by is largely unknown. Different surgical lead placements and different programming parameters are used. The relationship between lead placement, the electrical stimulation parameters, the perceived location of the ONS-induced supra-threshold sensations, the perceived spatial sensory field size, and the perceived sensory qualities with the reported clinical effectiveness are also largely unknown. For the analysis of this relationship, we used a computerbased imaging method [31] for mapping the ONSinduced perceived sensory location, the perceived 
spatial sensory field size, as well as the perceived sensory quality in a long-term course over 21 months in weekly time intervals. The aim of this study was to evaluate a potential relationship between supra-threshold-induced sensory perceptions and clinical effectiveness of ONS.

\section{METHODS}

\section{Compliance with Ethics Guidelines}

The ethics committee of the Medical Association of Schleswig-Holstein approved the study (FF11/2012). All subjects gave their informed written consent prior to participation. The study was performed in agreement with the Declaration of Helsinki.

\section{Subjects}

Thirty-two subjects treated with ONS (25 female and seven male, mean age $45.77 \pm 11.26$ years, age range, 22-67 years) were recruited prospectively at the Kiel Migraine and Headache Center. The subjects were all suffering from chronic migraine and were refractory to all other approved therapy options, which is why ONS was therapeutically indicated. Diagnosis and determination of indication was carried out by a minimum of two neurologists specialized in the field of headache therapy. Patients had a mean history of chronic migraine for $30.35 \pm 8.05$ years (range, $10-46$ years) and a mean history of 21 headache days/month (including both tension type headache and migraine days) before ONS implantation (range, 15-30 days/month). The MIDAS Score [32] before ONS implantation was evaluated for each patient to assess migraine disability. All subjects underwent implantation of the ONS device Eonmini $^{\mathrm{TM}}$ (St. Jude Medical Inc., USA) at the University Hospital Schleswig-Holstein (17 at campus Kiel, 15 at campus Lübeck). The mean follow-up time was $11.5 \pm 6.69$ months, range, 1-20.6 months. Only one patient was followed up for 1 month only, all other patients had a minimum follow-up of 4 months). The mean number of migraine and tension type headache days per month was continuously evaluated for each patient. During the whole observation period of 21 months, 808 individual weekly data sets of the relationship between the perceived stimulus location, electrical stimulation parameters, induced sensory qualities, and the individual clinical effect were recorded. On an individual basis, $24.28 \pm 20.29$ (range, 6-69) weekly data sets were collected.

\section{Surgical Technique}

The peripheral nerve stimulation systems used for occipital nerve stimulation for the treatment of chronic, intractable migraine consisted of an implantable pulse generator (IPG), which produces therapeutic electrical stimulation pulses. Two leads deliver the therapeutic stimulation pulses to the target location, each using eight electrode poles with $4 \mathrm{~mm}$ of distance in between. An external handheld device was used to adjust the intensity for each electrode pole for the stimulation. The leads were percutaneously implanted through a needle under general anesthesia. Once the lead was positioned in the desired location, it was anchored to the surrounding tissue to provide stability and avoid migration. Surgical implantation was carried out by two neurosurgical centers by two neurosurgeons. One center performed a trial period of 3 days after implantation to evaluate electrode positioning during this period. The other center implanted electrodes and IPG in one setting without a trial period. In this procedure, a pocket was created in the desired location in which the permanent IPG was stored. The IPG was implanted at the same anatomical location in both centers. Once the pocket was created, a subcutaneous path was created using a tunneling tool to allow the lead to be connected to the IPG. Once the lead was connected to the IPG and the system tested, the pocket was sutured and bandaged. After the permanent IPG was implanted, an external handheld device was used for programming. These devices were used by the clinician to select the stimulation parameters and by the patient to adjust the therapy levels to his/her individual comfort level. In this regard, no standardized requirements existed regarding 
individual electrical programming parameters (frequency, current strength, pulse width, and number and orientation of the electrode pole). Data collected were stored in the Relief register. Details about implantation and stimulation are described by Ashkan et al. [18].

\section{Data Collection}

After implantation, all subjects used a computer-based method for mapping spatial, cognitive and affective sensory effects of ONS (for details see [31]). This method enables continuous tracking of the ONS-induced perceived sensory location, the perceived spatial sensory field size, the perceived sensory quality and the rating of clinical effectiveness in the long term. Patients documented and rated location, intensity, quality and pleasantness of sensations, as well as headache days per month (see Fig. 1). Patients could additionally evaluate the general effectiveness of ONS therapy on a verbal rating scale. Thereby the categories "very good effect", "good effect", "moderate effect", "poor effect", "very poor effect", and "no effect" were available for selection. The rated intervals were classified as responder intervals, when the effectiveness was classified with "very good effect" up to "moderate effect". Non-responder intervals were defined as intervals with "poor effect" up to "no effect".

\section{Statistics}

Data were descriptively analyzed using IBM SPSS Statistics 24. Group comparisons of quantitative variables were carried out using the $t$ test. Relationships between categorical variables were analyzed using the Chi-square test and the Fisher-Yates test. The level of significance was set as $p \leq 0.05$.

\section{RESULTS}

\section{Rating of Effectiveness by the Patients}

Of the weekly stimulation intervals, $48.9 \%$ were classified as having had a "very good effect" up to "moderate effect" (so-called responders), $51.1 \%$ with "poor effect", "very poor effect" or "no effect" (so-called non-responders; Table 1). The responder rate in women was $52 \%$ and in men 33\% ( $p<0.0001$; Table 2$)$. The rating of effectiveness of ONS did not differ significantly depending on age at time of ONS implantation, the average number of migraine days per month, the MIDAS score, or the number of years suffering from migraine prior to the treatment (Table 3).

\section{Implantation with Trial Period vs. without Trial Period}

When electrodes and IPG were implanted in one surgical session without trial period, a responder rate of $55 \%$ could be observed. If after electrode implantation, a 3-day trial period was carried out before the IPG was permanently implanted, we found a significantly lower responder rate of $40 \%(p<0.0001$, Table 4$)$.

\section{Perceived Sensory Qualities}

The most frequently perceived sensory quality of "tingling" was significantly more frequent in non-responders than in responders $(p<0.0001$, Table 5). Perceived sensory qualities that patients reported to be slightly uncomfortable like humming, vibrating, pulsating, pinching, or knocking were more frequent in responders than in non-responders. Other than sensory quality, patients were also asked for sensory pleasantness (visual rating scale $1=$ "very pleasant" to 5 "very unpleasant"). In general, responders showed significantly higher pleasantness-scores than non-responders (pleasantness score of responders $3.01 \pm 0.81$; non-responders $2.23 \pm 1.01 ; p<0.0001)$.

\section{Perceived Sensory Location and Spatial Sensory Field Size}

To analyze the relationship between the spatial localization of sensations induced by ONS and the rating of effectiveness by the patients, we divided the occipital region into standardized areas (Fig. 1). For analysis, we drew a line 


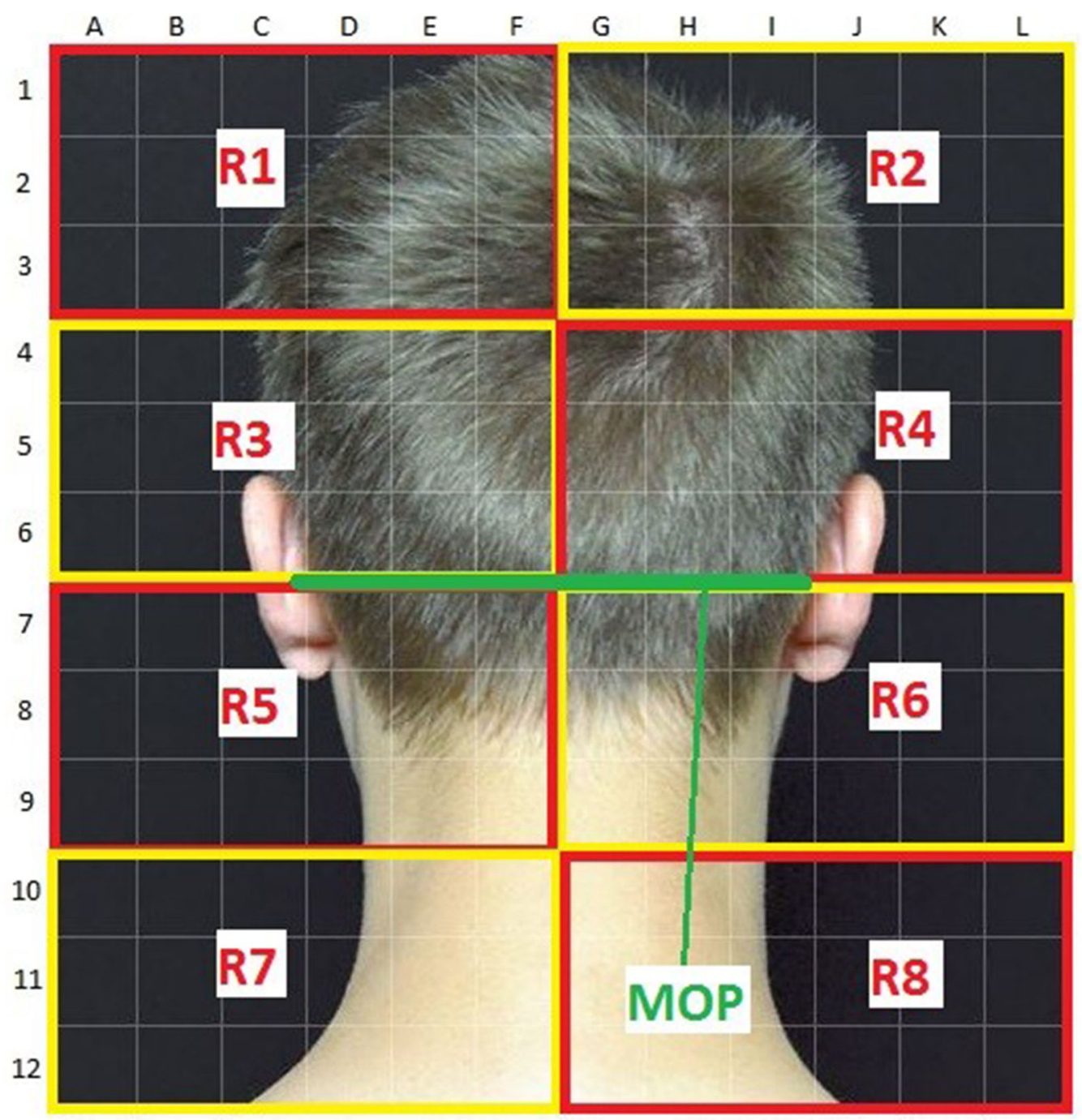

Fig. 1 Map of the back of the head with the line connecting the external acoustic meati and the external occipital protuberance (MOP line) highlighted in green. Above the MOP line are areas R1 to R4, below areas R5 to R8

Table 1 Evaluation of clinical effectiveness of ONS treatment by the patients

\begin{tabular}{lcl}
\hline Effectiveness rating & Number of intervals $(\boldsymbol{n}=\mathbf{8 0 8})$ & Proportion (\%) \\
\hline "Very good effect" & 96 & 11.88 \\
"Good effect" & 113 & 13.99 \\
"Moderate effect" & 186 & 23.02 \\
"Poor effect" & 207 & 25.62 \\
"Very poor effect" & 157 & 19.43 \\
"No effect" & 49 & 6.06 \\
\hline
\end{tabular}

The time intervals refer to the cumulated weekly time intervals during the treatment course 
Table 2 Rating of effectiveness of ONS treatment in by gender

\begin{tabular}{llllll}
\hline & Responder & Non-responder & Total & Chi $^{2}(\mathbf{1})$ & $\boldsymbol{p}$ \\
\hline Female & 343 & 308 & 651 & Chi $^{2}(1)=19.38$ & $<0.0001$ \\
Male & 52 & 105 & 157 & & \\
Total & 395 & 413 & 808 & & \\
\hline
\end{tabular}

The time intervals refer to the cumulated weekly time intervals during the treatment course. Responder $=$ interval rated as "very good effect" to "moderate effect". Non-responder = interval rated as "poor effect" to "no effect"

Table 3 Rating of effectiveness of ONS treatment as a function of patient age at time of ONS implantation, the average number of migraine days per month, the MIDAS score, and the years of migraine

disorder prior to ONS treatment ( $n$ number, SD standard deviation, $t t$ test, $d f$ degrees of freedom, $p$ represents the value of probability)

\begin{tabular}{llllllll}
\hline & Effectiveness & $\boldsymbol{n}$ & Average & SD & $\boldsymbol{t}$ & $\boldsymbol{d} f$ & $\boldsymbol{p}$ \\
\hline Age at time of ONS implantation (years) & Responder & 375 & 48.47 & 10.946 & 0.625 & 773 & 0.531 \\
& Non-responder & 400 & 314 & 21.68 & & & \\
Average number of migraine days per month & Responder & 314 & 21.68 & 5.876 & 1.725 & 663 & 0.085 \\
& Non-responder & 351 & 22.53 & 6.735 & & & \\
MIDAS score at treatment start & Responder & 369 & 124.55 & 65.200 & 1.542 & 764 & 0.123 \\
& Non-responder & 397 & 131.70 & 63.068 & & & \\
Years of migraine prior to ONS treatment & Responder & 305 & 30.30 & 12.129 & 0.473 & 643 & 0.636 \\
& Non-responder & 340 & 29.80 & 14.409 & & & \\
\hline
\end{tabular}

Table 4 Effect of ONS implantation with trial period versus without trial period

\begin{tabular}{llllll}
\hline & Responder & Non-responder & Total & Chi $^{\mathbf{2}}(\mathbf{1})$ & $\boldsymbol{p}$ \\
\hline Without trial period & 257 & 209 & 466 & 17.29 & $<0.0001$ \\
With trial period & 138 & 204 & 342 & & \\
Total & 306 & 281 & 808 & & \\
\hline
\end{tabular}

between the external acoustic meatus and the external occipital protuberance (MOP line) and analyzed the effect of induced sensations in the areas both below and above this line (the MOP line highlighted in green is shown in Fig. 1). The area above the MOP line was divided into areas $\mathrm{R} 1$ to $\mathrm{R} 4$ and the area below the MOP line as divided into areas R5 to R8.

Sensations that were spatially perceived above the MOP line led to patients reporting a positive clinical effect significantly more frequently than sensations spatially perceived below the MOP line (Table 6). The side of stimulus perception (right versus left) did not have a significant influence on the reporting of effectiveness. A spatially small fields of sensory perception with a small number of stimulated single quadrants above the MOP line was associated with a higher probability of clinical effectiveness than multiple single quadrants covering a broader field of perception $(p<0.0001$, Fig. 2 and examples in Fig. 3 ). 
Table 5 Perceived sensory qualities in relation to the reported clinical effectiveness of ONS

\begin{tabular}{lcccc}
\hline & Responder & Non-responder & Chi $^{\mathbf{2}}$ (6) & P \\
\hline Knocking & 4 & 3 & 37.88 & \\
Tingling & 247 & 334 & & \\
Pulsating & 3 & 4 & & \\
Throbbing & 15 & 6 & \\
Humming & 15 & 12 & \\
Vibrating & 36 & 13 & \\
Pinching & 75 & 41 & \\
Total & 395 & 413 & \\
\hline
\end{tabular}

Table 6 Number of the areas stimulated above and below the MOP line out of R1 to R8 in relation to the reported clinical effectiveness ( $n$ number, $S D$ standard deviation, $t t$ test, $d f$ degrees of freedom, $p$ represents the value of probability)

\begin{tabular}{|c|c|c|c|c|c|c|c|}
\hline Area with perceived sensation & Effectiveness & $n$ & Average & SD & $t$ & $d f$ & $p$ \\
\hline \multirow[t]{2}{*}{ R1 } & Responder & 395 & 0.12 & 0.541 & -8.71 & 806 & $<0.0001$ \\
\hline & Non-responder & 413 & 0.73 & 1.299 & -8.85 & 556 & $<0.0001$ \\
\hline \multirow[t]{2}{*}{ R2 } & Responder & 395 & 0.09 & 0.523 & -9.60 & 806 & $<0.0001$ \\
\hline & Non-responder & 413 & 0.78 & 1.347 & -9.76 & 538 & $<0.0001$ \\
\hline \multirow[t]{2}{*}{ R3 } & Responder & 395 & 1.68 & 1.919 & -11.53 & 806 & $<0.0001$ \\
\hline & Non-responder & 413 & 3.91 & 3.364 & -11.66 & 660 & $<0.0001$ \\
\hline \multirow[t]{2}{*}{$\mathrm{R} 4$} & Responder & 395 & 1.46 & 1.670 & -12.14 & 806 & $<0.0001$ \\
\hline & Non-responder & 413 & 3.77 & 3.413 & -12.32 & 604 & $<0.0001$ \\
\hline \multirow[t]{2}{*}{ R5 } & Responder & 395 & 1.29 & 1.383 & 0.95 & 806 & 0.34 \\
\hline & Non-responder & 413 & 1.20 & 1.452 & 0.95 & 805 & 0.34 \\
\hline \multirow[t]{2}{*}{ R6 } & Responder & 395 & 1.27 & 1.461 & 0.93 & 806 & 0.35 \\
\hline & Non-responder & 413 & 1.17 & 1.570 & 0.93 & 805 & 0.35 \\
\hline \multirow[t]{2}{*}{ R7 } & Responder & 395 & 0.06 & 0.386 & 0.92 & 806 & 0.35 \\
\hline & Non-responder & 413 & 0.03 & 0.277 & 0.02 & 711 & 0.36 \\
\hline \multirow[t]{2}{*}{ R8 } & Responder & 395 & 0.05 & 0.367 & 1.29 & 806 & 0.20 \\
\hline & Non-responder & 413 & 0.02 & 0.183 & 1.27 & 573 & 0.20 \\
\hline
\end{tabular}

\section{DISCUSSION}

In this study, we examined, to our knowledge, for the first time a possible relationship in supra-threshold sensory perception between perceived sensory quality, perceived sensory location, perceived spatial field size, and individual clinical effect in the treatment of chronic migraine with occipital nerve stimulation in the long term. It remains unclear whether occipital 


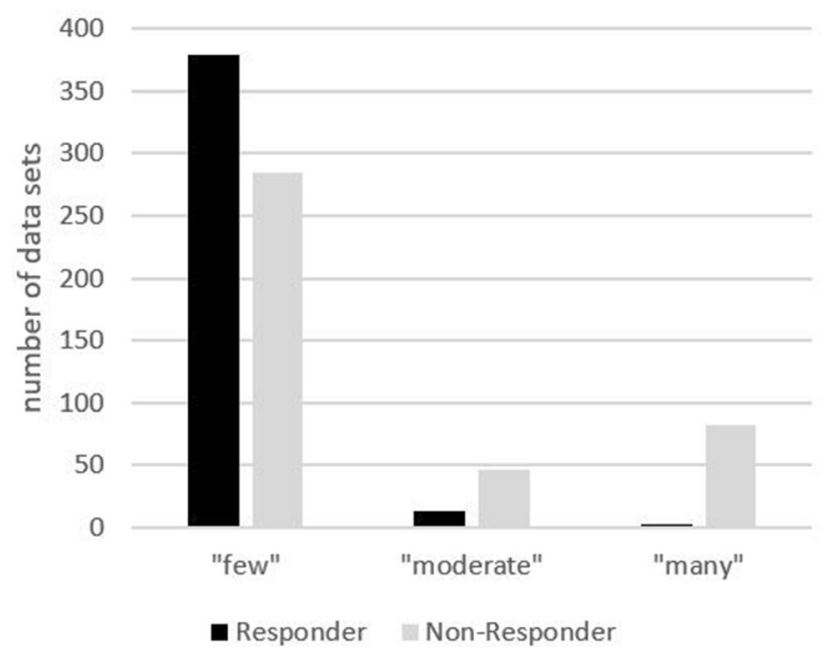

Fig. 2 Number of stimulated single quadrants above the MOP line as a function of rating of clinical effectiveness (categories: $0-10$ fields $=$ "few", 11-19 fields = "moderate", 20-29 fields = "many")

nerve stimulation is also clinically effective in sub-threshold sensory perception. Evidence for the latter comes from an open pilot study with bilateral burst pattern ONS [19]. Of the weekly stimulation intervals analyzed in this study, $48.9 \%$ were rated as very good, good, or moderate regarding the effectiveness of ONS. Neither age, the average number of migraine days per month, the MIDAS score, or the duration of the migraine disorder prior to ONS treatment showed a relationship with the reported effectiveness of the therapy. Patients in whom the electrodes and the IPG was fully implanted in one session without a subsequent trial period showed a significantly higher responder rate than patients, in which a trial period took place. This provides arguments against the necessity of a trial period. We are not aware of studies that directly compared an implantation with or without a trial period. Our data indicate that direct implantation can lead to more effective results. It must remain open at present whether altered scar formation or contact conditions are responsible or whether other reasons exist.

The most frequently reported sensation was that of "tingling". This sensation is however a significant predictor for a lower effectiveness and should therefore be avoided in programming of stimulation parameters. More aversive sensory qualities that are slightly uncomfortable, like humming, vibrating, pulsating, pinching, or knocking are more frequent in responders than in non-responders. We recommend to individually select electrical parameters during programming that induce such sensations to more likely achieve a higher clinical effectiveness using ONS. It can be speculated that through a low sustained nociceptive stimulation, the antinociceptive system is activated, leading to a desensitization $[1,9,22$, 30, 33, 34].

A horizontal or vertical electrode position relative to the course of the occipital nerve as well as the height of the location is done differently by different centers. Sensations that were perceived in location above the line connecting the external acoustic meati and the external occipital protuberance (MOP line) significantly more frequently led to patients reporting a positive clinical effect. This implies that electrodes should be placed above the MOP line for ONS to achieve a higher clinical effectiveness. Not just the location seems to be of importance here. The two electrodes have eight active poles each that can be individually programmed. Induced sensations covering broader areas in the innervation areas of the $\mathrm{C} 2$ and $\mathrm{C} 3$ dermatomes led to a lesser clinical effect according to our data. Sensations that are induced in a spatially concentrated field in the area of the external occipital protuberance on the other hand increase the probability of 

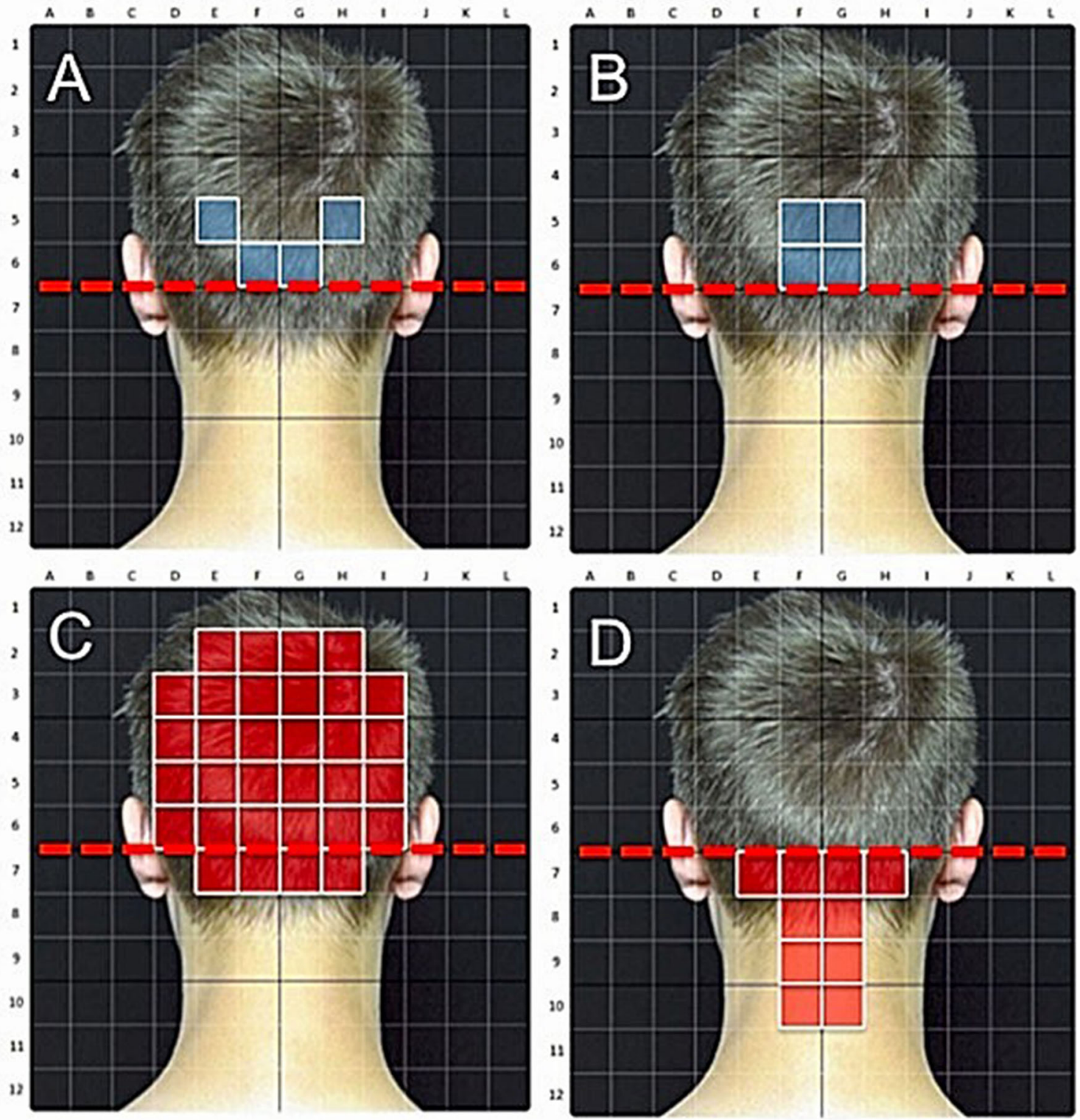

Fig. 3 a, b Exemplary spatial distribution of induced sensations leading to effective ONS treatment rating by the patient. c, d Exemplary spatial distribution of induced sensations leading to ineffective ONS treatment rating by the patient

clinical effectiveness (see examples in Fig. 3). A narrow " $\mathrm{Y}$ "-shaped electrode positioning close to the midline therefore appears to be more favorable than a wide laterally spaced-out " $\mathrm{T}$ "shaped one. Additionally, the individual active electrode poles should be programmed in such a way that a sensory perception in a spatially small perceptive field close to the midline and above the occipital protuberance over the main branches of the occipital nerve results. We assume that a corresponding " $\mathrm{Y}$ "-shaped electrode positioning and programming determines this sensory perception. Because the exact electrode positioning was not documented for all patients, we can make this statement only for the induced sensory pattern.

When programming the IPG for occipital nerve stimulation, electric current, frequency, phase width and number, as well as orientation of electrode poles can be specified. No standard 
has yet been established on how these parameters should be programmed. Our data indicate that induced sensations can change over time using the same set of stimulation parameters. This may on the one hand be based on neuronal adaptation and habituation processes. On the other hand, several other local factors may modulate the sensory effects, such as local impedances, relation of the electrode to the fascia, and possible unintended direct contact of the electrode to nerve structures. Also, local scarring and small electrode movements may be of relevance. For these reasons, an individual and continuous (re-)programming of the impulse generator in the long term is generally necessary. A generic electrical impulse pattern is thereby not feasible because this leads to completely different sensory perception patterns due to the aforementioned reasons.

This study was not designed to confirm the efficacy of ONS in chronic migraine. Neuromodulation may be beneficial in a subgroup of patients with intractable chronic migraine. The aim was to analyze a potential relationship between electrical and sensory parameters in the supra-threshold sensory perception range with clinical effectiveness. Awareness of this relationship could improve the efficacy of ONS for the treatment of chronic migraine and may in part explain the different intraindividual and interindividual outcomes in previous clinical studies of ONS in the treatment of migraine $[1,3,4,7-11,13,14,17-26,28,34-36]$.

\section{CONCLUSIONS}

The ONS-induced sensory location, the size of the spatial sensory field, as well as the sensory quality are significantly correlated with the reported clinical effectiveness. The results suggest that besides surgical technique, the individual and continuous programming of the stimulation parameters is important in increasing the therapeutic effectiveness. Further studies are necessary to uncover the physiological, psychophysical, and perceptional-psychological mechanisms.

\section{ACKNOWLEDGEMENTS}

We thank the participants of the study.

Funding. No funding or sponsorship was received for this study or publication of this article.

Authorship. All named authors meet the International Committee of Medical Journal Editors (ICMJE) criteria for authorship for this article, take responsibility for the integrity of the work as a whole, and have given their approval for this version to be published.

Authorship Contributions. Carl Göbel: design and conceptualization of the study, interpretation of the results, contributed to statistical analysis, writing of the first draft of the manuscript. Anna Göbel: interpretation of the results and contribution to analysis of data. Uwe Niederberger: statistical analysis. Axel Heinze: contribution to interpretation and analysis. Katja Heinze-Kuhn: contribution to interpretation and analysis. Christoph Meinecke: contribution to interpretation and analysis. Dirk Rasche: contribution to interpretation and analysis. Hubertus H. Mehdorn: contribution to interpretation and analysis. Hartmut Göbel: design and conceptualization of the study, co-writing of the manuscript.

Disclosures. Carl H. Göbel, Anna Göbel, Uwe Niederberger, Axel Heinze, Katja HeinzeKuhn, Christoph Meinecke, Hubertus M. Mehdorn, Dirk Rasche and Hartmut Göbel have nothing to disclose.

Compliance with Ethics Guidelines. The ethics committee of the Medical Association of Schleswig-Holstein approved the study (FF11/ 2012). All subjects gave their informed written consent prior to participation. The study was performed in agreement with the Declaration of Helsinki.

Data Availability. The datasets generated during and/or analyzed during the current study are available from the corresponding author on reasonable request. 
Open Access. This article is licensed under a Creative Commons Attribution-NonCommercial 4.0 International License, which permits any non-commercial use, sharing, adaptation, distribution and reproduction in any medium or format, as long as you give appropriate credit to the original author(s) and the source, provide a link to the Creative Commons licence, and indicate if changes were made. The images or other third party material in this article are included in the article's Creative Commons licence, unless indicated otherwise in a credit line to the material. If material is not included in the article's Creative Commons licence and your intended use is not permitted by statutory regulation or exceeds the permitted use, you will need to obtain permission directly from the copyright holder. To view a copy of this licence, visit http://creativecommons.org/licenses/by$\mathrm{nc} / 4.0 /$.

\section{REFERENCES}

1. Chen YF, Bramley G, Unwin G, Hanu-Cernat D, Dretzke J, Moore D, et al. Occipital nerve stimulation for chronic migraine-a systematic review and meta-analysis. PLoS ONE. 2015;10(3):e0116786.

2. Ashkan K, Dowson A. Peripheral occipital nerve stimulation to treat chronic refractory migraine. $\mathrm{Br}$ J Hosp Med. 2013;74(2):85-9.

3. Brewer AC, Trentman TL, Ivancic MG, Vargas BB, Rebecca AM, Zimmerman RS, et al. Long-term outcome in occipital nerve stimulation patients with medically intractable primary headache disorders. Neuromodulation J Int Neuromodulation Soc. $2013 ; 16(6): 557-64$.

4. Fontaine D, Christophe Sol J, Raoul S, Fabre N, Geraud G, Magne C, et al. Treatment of refractory chronic cluster headache by chronic occipital nerve stimulation. Cephalalgia Int $\mathrm{J}$ Headache. 2011;31(10):1101-5.

5. Goadsby PJ. Analysis of occipital nerve stimulation in studies of chronic migraine and broader implications of social media in clinical trials. Cephalalgia Int J Headache. 2013;33(3):214-5.

6. Magis D, Gerardy PY, Remacle JM, Schoenen J. Sustained effectiveness of occipital nerve stimulation in drug-resistant chronic cluster headache. Headache. 2011;51(8):1191-201.
7. Saper JR, Dodick DW, Silberstein SD, McCarville S, Sun M, Goadsby PJ, et al. Occipital nerve stimulation for the treatment of intractable chronic migraine headache: ONSTIM feasibility study. Cephalalgia Int J Headache. 2011;31(3):271-85.

8. Schwedt TJ. Occipital nerve stimulation for chronic migraine-interpreting the ONSTIM feasibility trial. Cephalalgia Int J Headache. 2011;31(3):262-3.

9. Schwedt TJ, Vargas B. Neurostimulation for treatment of migraine and cluster headache. Pain Med. 2015;16(9):1827-34.

10. Serra G, Marchioretto F. Occipital nerve stimulation for chronic migraine: a randomized trial. Pain Phys. 2012;15(3):245-53.

11. Silberstein SD, Dodick DW, Saper J, Huh B, Slavin $\mathrm{KV}$, Sharan A, et al. Safety and efficacy of peripheral nerve stimulation of the occipital nerves for the management of chronic migraine: results from a randomized, multicenter, double-blinded, controlled study. Cephalalgia Int J Headache. 2012;32(16):1165-79.

12. Strand NH, Trentman TL, Vargas BB, Dodick DW. Occipital nerve stimulation with the Bion(R) microstimulator for the treatment of medically refractory chronic cluster headache. Pain Phys. 2011;14(5):435-40.

13. Tronnier V, Rasche D. Subcutaneous peripheral stimulation of the greater occipital nerve for the treatment of chronic headache syndromes. Schmerz. 2010;24(5):441-8.

14. Wilbrink LA, Teernstra OP, Haan J, van Zwet EW, Evers SM, Spincemaille GH, et al. Occipital nerve stimulation in medically intractable, chronic cluster headache. The ICON study: rationale and protocol of a randomised trial. Cephalalgia Int J Headache. 2013;33(15):1238-47.

15. Young WB. Occipital nerve stimulation for chronic migraine. Curr Pain Headache Rep. 2014;18(2):396.

16. Young WB, Silberstein SD. Occipital nerve stimulation for primary headaches. J Neurosurg Sci. 2012;56(4):307-12.

17. Yang Y, Song M, Fan Y, Ma K. Occipital nerve stimulation for migraine: a systematic review. Pain Pract Off J World Inst Pain. 2015;16(4):509-17.

18. Ashkan K, Sokratous G, Gobel H, Mehta V, Gendolla A, Dowson A et al. Peripheral nerve stimulation registry for intractable migraine headache (RELIEF): a real-life perspective on the utility of occipital nerve stimulation for chronic migraine. Acta Neurochir 2020. 
19. Garcia-Ortega R, Edwards T, Moir L, Aziz TZ, Green AL, FitzGerald JJ. Burst occipital nerve stimulation for chronic migraine and chronic cluster headache. Neuromodulation J Int Neuromodulation Soc. 2019;22(5):638-44.

20. Rodrigo D, Acin P, Bermejo P. Occipital nerve stimulation for refractory chronic migraine: results of a long-term prospective study. Pain Phys. 2017;20(1):E151-E159159.

21. Liu Y, Dong Z, Wang R, Ao R, Han X, Tang W, et al. Migraine prevention using different frequencies of transcutaneous occipital nerve stimulation: a randomized controlled trial. J Pain. 2017;18(8): 1006-155.

22. Yang Y, Song M, Fan Y, Ma K. Occipital nerve stimulation for migraine: a systematic review. Pain Pract Off J World Inst Pain. 2016;16(4):509-17.

23. Miller S, Watkins L, Matharu M. Long-term outcomes of occipital nerve stimulation for chronic migraine: a cohort of 53 patients. J Headache Pain. 2016;17(1):68.

24. Schwedt TJ, Green AL, Dodick DW. Occipital nerve stimulation for migraine: update from recent multicenter trials. Prog Neurol Surg. 2015;29:117-26.

25. Dodick DW, Silberstein SD, Reed KL, Deer TR, Slavin KV, Huh B, et al. Safety and efficacy of peripheral nerve stimulation of the occipital nerves for the management of chronic migraine: long-term results from a randomized, multicenter, double-blinded, controlled study. Cephalalgia Int $\mathrm{J}$ Headache. 2015;35(4):344-58.

26. Palmisani S, Al-Kaisy A, Arcioni R, Smith T, Negro A, Lambru G, et al. A six-year retrospective review of occipital nerve stimulation practice-controversies and challenges of an emerging technique for treating refractory headache syndromes. J Headache Pain. 2013;14:67.

27. Franzini A, Messina G. Surgery for treatment of refractory chronic cluster headache: toward standard procedures. Neurol Sci Off J Ital Neurol Soc Ital Soc Clin Neurophys. 2015;36(Suppl 1):131-5.

28. Slotty PJ, Bara G, Vesper J. The surgical technique of occipital nerve stimulation. Acta Neurochir. 2015;157(1):105-8.
29. Trentman TL, Zimmerman RS, Dodick DW. Occipital nerve stimulation: technical and surgical aspects of implantation. Prog Neurol Surg. 2011;24: 96-108.

30. Walling I, Smith H, Gee LE, Kaszuba B, Chockalingam A, Barborica A, et al. Occipital nerve stimulation attenuates neuronal firing response to mechanical stimuli in the ventral posteromedial thalamus of a rodent model of chronic migraine. Neurosurgery. 2017;81(4):696-701.

31. Gobel A, Gobel CH, Heinze A, Heinze-Kuhn K, Petersen I, Meinecke C, et al. Imaging sensory effects of occipital nerve stimulation: a new computer-based method in neuromodulation. Brain Stimul. 2015;8(2):295-8.

32. Stewart WF, Lipton RB, Whyte J, Dowson A, Kolodner K, Liberman JN, et al. An international study to assess reliability of the Migraine Disability Assessment (MIDAS) score. Neurology. 1999;53(5): 988-94.

33. De La Cruz P, Gee L, Walling I, Morris B, Chen N, Kumar V, et al. Treatment of allodynia by occipital nerve stimulation in chronic migraine rodent. Neurosurgery. 2015;77(3):479-85.

34. Jenkins B, Tepper SJ. Neurostimulation for primary headache disorders, part 1: pathophysiology and anatomy, history of neuromodulation in headache treatment, and review of peripheral neuromodulation in primary headaches. Headache. 2011;51(8): 1254-66.

35. Lipton RB, Goadsby P, Cady R, Aurora S, Grosberg B, Freitag F, Silberstein S, Whiten D, Jaax K, et al. PRISM study: occipital nerve stimulation for treatment-refractory migraine. Headache. 2010;50(3): 509-19.

36. Clark SW, Wu C, Boorman DW, Chalouhi N, Zanaty $M$, Oshinsky $M$, et al. Long-term pain reduction does not imply improved functional outcome in patients treated with combined supraorbital and occipital nerve stimulation for chronic migraine. Neuromodulation J Int Neuromodulation Soc. 2016;19(5):507-14. 